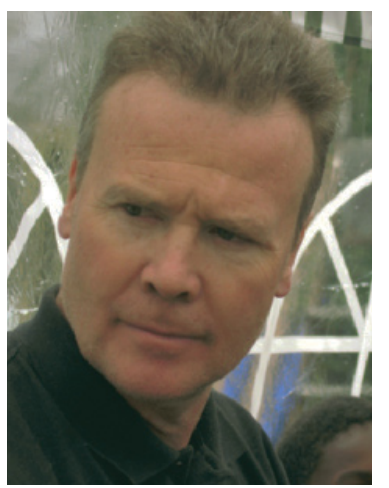

G. Dine

\section{Recherche translationnelle et biologie clinique}

L'émergence de la génomique et de la protéomique a considérablement changé l'organisation de la recherche industrielle du secteur pharmaceutique. L'utilisation conjointe des outils scientifiques et technologiques relevant des sciences du vivant, de la santé mais aussi des sciences de l'ingénieur a permis l'accélération conséquente des processus d'innovation en vue d'obtenir des produits thérapeutiques efficaces.

Vu la multiplicité des données à traiter et la complexicité des tâches à accomplir, il fallait une interface indispensable identifiée sous le terme recherche translationnelle. La relation rapide entre amont scientifique et application clinique est devenue en effet un espace stratégique pour les entreprises de santé. II est parfaitement défini dans le langage de nos collègues nord-américains : From bench to bed.

Les laboratoires pharmaceutiques font largement appel aujourd'hui à ce mode organisationnel centré autour de la recherche translationnelle qui intègre les aspects cliniques incontournables.

La mise à disposition ces dernières années de thérapies ciblées très efficaces grâce à la fabrication de protéines recombinantes, d'anticorps monoclonaux, de petites molécules de synthèse correspond à cette nouvelle donne.

Le secteur de la biologie clinique n'échappe à cette tendance lourde. Pour relever ce challenge les entreprises doivent gérer de façon simultanée l'ensemble des éléments indispensables à l'innovation ce qui sous entend la mise en place de partenariats multiples souvent publiques-privés et une politique d'interface scientifique et technologique faisant appel à de nombreuses compétences y compris les services d'hospitalisation et les laboratoires nécessaires à la recherche clinique sous réserve que ces unités disposent d'un environnement adapté échappant aux freins administratifs d'organismes seulement centrés sur la problématique des soins.

Les principes qui régissent le management de l'innovation tel qu'il est pratiqué dans des domaines technologiques pointus comme l'aéronautique et l'énergie sont aujourd'hui employés par les entreprises de santé qu'elles soient pharmaceutiques ou biomédicales.

Ce numéro n'est pas thématique. II présente plusieurs articles proposés par différents auteurs à la publication qui témoignent de cet état d'esprit nouveau basé sur la mise en interaction ordonnée de savoirs faire complémentaires appliqués au diagnostic in vitro.

En exergue des thèmes scientifiques et technologiques traités par ces articles, le compte-rendu du dernier Café Scientifique réalisé par la revue à l'occasion des Journées Internationales de Biologie en novembre 2009 témoigne de l'intérêt de cette démarche industrielle vis-à-vis de la production de tests biologiques performants qui modifient la prise en charge diagnostique et thérapeutique proposée aux patients concernés par certaines maladies. 